the same patient were consistent when repeated. In comparison with the macrophages in Hodgkin's disease, those in sarcoma showed significantly more multinucleated forms and the maximum number of nuclei seen in a single cell was higher. In a single patient with lymphosarcoma, macrophage mitosis was noted, but has not been found in any other patient even when colcemid has been applied locally.

These changes may suggest a disordered cellular response.

\section{Serum Creatine Phosphokinase Changes in} Psychotic Illness

G. OWEN, R. GOSLING, AND R. J. KERRY (Northern General Hospital, Sheffield)

Increased serum creatine phosphokinase (CPK) levels have been reported in acutely psychotic patients. This study examined the proportion of psychotic and non-psychotic patients showing raised serum CPK activity. This occurred in about half of the patients with psychotic illnesses but was absent in the nonpsychotic patients. Raised serum CPK was significantly associated with second order factor score patterns on the Inpatient Multiphasic Psychiatric Scale (IMPS) suggesting that mania and paranoid schizophrenia were more involved with this enzyme change than depression and non-paranoid schizophrenia.

Raised serum CPK suggests that psychotic illness may be present. If a patient is found to have an increased level of CPK, in the absence of organic illness, further psychiatric investigation is required. Increased CPK activity may precede the clinical manifestations of the psychotic illness by a few days. This has been seen in the symptom-free outpatients with a raised CPK who have been admitted to hospital a few days later with acute psychotic illness. Creatine phosphokinase estimation would appear to be a worthwhile screening procedure for both inpatients and outpatients. Patients with raised CPK need continued psychiatric supervision.

\section{The Incidence of Syphilis in Thailand}

P. SONGHAPRASERT, B. RUNGPITARANGSI, AND S. PHANSOMBOON (Siriraj Hospital, Bangkok, Thailand)

Serological investigations for syphilis, carried out on specimens collected as a routine from Bangkok hospital out- patients, gave positive results in $7.1 \%$ of cases. This suggests the high incidence of syphilis of 7100 per 100000 population.

\section{Management of Cases of Myelomatosis E. WILSHAW (Royal Marsden Hospital, London)}

The malignant proliferation of plasma cells exposes a patient to certain hazards which must be dealt with adequately if he is to benefit from any remission achieved by chemotherapy.

BONE LESIONS

Solitary filling defects are best treated with large doses of radiotherapy with a view to cure. Large osteolytic areas in limbs should be supported by pinning since recalcification of myelomatosis lesions is very rare. Paraplegia must be treated early, preferably by laminectomy followed by radiotherapy. Hypercalcaemia is treated with prednisone and neutral phosphate together with alkylating agents. Patients should be mobilized as soon as possible.

\section{PARAPROTEIN PRODUCTION}

Hyperviscosity can be alleviated temporarily by plasmaphoresis while giving time for the alkylating agents to reduce the mass of myeloma. Amyloid deposition is irreversible but the carpal tunnel syndrome can be relieved by surgical excision of the retinaculum.

\section{BONE MARROW FUNCTION}

The ability to make normal numbers of erythrocytes, leucocytes, and platelets can be restored by alkylating agents provided that the treatment is given persistently. Rises in haemaglobin may be seen only when treatment has been given for a year.

\section{Factors of Prognostic Significance in} Myelomatosis

R. PETO (Radcliffe Infirmary, Oxford)

In 1964 the MRC initiated a clinical trial which compared two protocols for the treatment of myelomatosis. Nearly 300 patients were notified by 1968 , and by 1971 all these patients had been at risk for at least three years. Because the protocols proved therapeutically identical, this series was ideal for assessing the influence on subsequent prognosis of the initial condition of a myelomatosis patient. Features such as hypercalcaemia, which are corrected by the MRC protocol, do not appear in this series to influence prognosis. Conversely, renal failure is ir- relevant to the neoplastic disease but, being irreversible by the MRC protocols, dominates prognosis so strongly that a patient presenting with a blood urea above $80 \mathrm{mg} / 100 \mathrm{ml}$ is likely to be dead within two months, whereas one presenting with a blood urea below $40 \mathrm{mg} / 100 \mathrm{ml}$ is likely to live over three years. To study the actual myeloma we therefore need to find what, given the effect of, renal failure on prognosis, is the effect on prognosis of the various biochemical, histological, haematological, and radiological measurements made in the MRC trial as the patients were notified. We found to our surprise that although many measurements were abnormal, few of these abnormalities mattered. None of the radiological, histological, haematological (other than anaemia), or biochemical (other than hypoalbuminaemia) measurements mattered at all (except insofar as they correlated with renal failure), and the series is so large that this is quite a definite conclusion. We do not really know why anaemia matters. Perhaps it is a measure of marrow displacement, but then why do low platelet or neutrophil counts not matter at all? We have still less idea why hypoalbuminaemia matters. In experimental animals, some tumours can concentrate labelled albumin in them and cause hypoalbuminaemia, so perhaps the more active myelomas catabolize circulating albumin.

The Clinical Significance of Bence Jones Proteinuria

H. MCLAUGHLIN (Westminster Hospital Medical School, London)

Bence Jones protein is monoclonal light chain, found in the urine because of its low molecular weight $(22000)$. Monoclonality is demonstrated by cellulose acetate electrophoresis of concentrated urine in parallel with serum. Immunoelectrophoresis will identify the type of $\mathbf{L}$ chain involved. Free light chain will react with its corresponding antiserum across $3 \mathrm{~mm}$ agar within two hours, light chain bound to IgG takes three hours, and that bound to IgM eight to 12 hours.

Concentration of urine up to $\times 300$ is essential to exclude low levels of Bence Jones protein and this is best done by vacuum dialysis in collodion thimbles of urine, initially passed through $1.0 \mu$ and $0 \cdot 2 \mu$ filters to remove bacteria.

The Table shows the results when this technique was used in 1159 cases of various disorders. 